Review

\title{
Narrow band imaging-assisted transurethral resection reduces the recurrence risk of non-muscle invasive bladder cancer: A systematic review and meta-analysis
}

\author{
Weiting Kang ${ }^{1,3, *}$, Zilian Cui ${ }^{1,3, *}$, Qianqian Chen ${ }^{2,3}$, Dong Zhang ${ }^{1}$, Haiyang Zhang ${ }^{1}$ \\ and Xunbo Jin ${ }^{1}$ \\ ${ }^{1}$ Minimally Invasive Urology Center, Shandong Provincial Hospital Affiliated to Shandong University, Jinan, Shandong, China \\ 2 Department of Emergency, Shandong Provincial Hospital Affiliated to Shandong University, Jinan, Shandong, China \\ ${ }^{3}$ School of Medicine, Shandong University, Jinan, Shandong, China \\ * These authors have contributed equally to this work \\ Correspondence to: Xunbo Jin, email: jinxunbo@163.com \\ Zilian Cui, email: 122526195@qq.com \\ Keywords: non-muscle invasive bladder cancer; narrow band imaging; white light imaging; resection; recurrence risk \\ Received: August 27, $2016 \quad$ Accepted: October 27, $2016 \quad$ Published: November 03, 2016
}

\section{ABSTRACT}

Context: Compared with white light imaging (WLI) cystoscopy, narrow band imaging (NBI) cystoscopy could increase the visualization and detection of bladder cancer (BC) at the time of transurethral resection (TUR). NBI cystoscopy could increase the detection of $\mathrm{BC}$, but it remains unclear whether narrow band imagingassisted transurethral resection (NBI-TUR) could reduce the recurrence risk of nonmuscle invasive bladder cancer (NMIBC). Several randomized clinical trials (RCTs) have recently tested the efficacy of NBI-TUR for NMIBC.

Objective: To perform a systematic review and meta-analysis of RCTs and evaluate the efficacy of NBI-TUR for NMIBC compared with white light imagingassisted transurethral resection (WLI-TUR). The end point was recurrence risk.

Evidence acquisition: A systematic review of PubMed, Medline, Ovid, Embase, Cochrane and Web of Science was performed in February 2016 and updated in July 2016.

Evidence synthesis: Overall, six $(n=1084)$ of 278 trials were included. Three trials performed narrow band imaging-assisted electro-transurethral resection (NBIETUR), and two trials performed narrow band imaging-associated bipolar plasma vaporization (NBI-BPV). The last trial performed narrow band imaging-associated holmium laser resection (NBI-HLR). Statistical analysis was performed using Review Manager software (RevMan v.5.3; The Nordic Cochrane Center, Copenhagen, Denmark). The recurrence risk was compared by calculating risk ratios (RRs) with 95\% confidence interval (CIs). Risk ratios with 95\% CIs were calculated to compare 3-mo, 1-yr, and 2-yr survival rates. NBI-TUR was associated with improvements in the 3-mo recurrence risk (RR: 0.39; 95\% CI, 0.26-0.60; $p<0.0001$ ), 1-yr recurrence risk (RR: $0.52 ; 95 \% \mathrm{CI}, 0.40-0.67 ; p<0.00001$ ) and 2-yr recurrence risk (RR: 0.60 ; 95\% CI, 0.42-0.85; $p=0.004$ ) compared with WLI-TUR.

Conclusions: Compared with WLI-TUR, NBI-TUR can reduce the recurrence risk of NMIBC. The results of this review will facilitate the appropriate application of NBI in NMIBC. 


\section{INTRODUCTION}

Bladder cancer $(\mathrm{BC})$ is a heterogeneous disease and the fourth most common malignant tumor, after prostate cancer, lung cancer, and colon cancer, in Western countries [1]. The incidence of $\mathrm{BC}$ is three to four times higher in men than in women. In the European Union, the incidence is 27 in 100000 for men and 6 in 100000 for women [2]. Fortunately, most newly diagnosed BCs are non-invasive urothelial tumors that are confined to the mucosa or mucosal lamina propria [3]. However, it is a long-term process from a predisposing change to relapse [4]. Although most poorly differentiated BCs do not progress, up to $20 \%$ of non-muscular infiltrating tumors can progress into myometrial invasion or metastasis [5]. It is a major challenge to reduce the high frequency of early recurrence risk of non-muscle invasive bladder cancer (NMIBC) because of the high recurrence rate, which can be as high as $45 \%$ at the first follow-up cystoscopy, 3 mo after TUR [6]. Bladder cancer recurrence and progression differ greatly with respect to tumor multiplicity, size, previous recurrence rates, $\mathrm{T}$ category, presence of carcinoma in situ (CIS) and grade [7]. In fact, most early "recurrences" are overlooked or residual tumors, and thus, it is important to increase $\mathrm{BC}$ visualization and detection for NMIBC. Neglected lesions will significantly affect the patient's management and outcome [8].

Micro-papillary or early flat in situ carcinomas can be difficult to detect by white light imaging (WLI) cystoscopy, but narrow band imaging (NBI) cystoscopy can improve the detection rate of recurrent flat and papillary superficial BC [9]. Herr and Donat evaluated recurrent BCs by WLI cystoscopy, followed by NBI cystoscopy, and found that $24 \%$ of patients had recurring cancer; $87 \%$ were detected by both WLI and NBI, and $100 \%$ were detected only by NBI cystoscopy. NBI cystoscopy can detect more papillary tumors or carcinoma in situ in $56 \%$ of recurrent patients [9]. NBI is an optical enhancement technology comprising two bandwidths of illumination centered on blue $(415 \mathrm{~nm})$ and green $(540 \mathrm{~nm})$. NBI can increase the contrast between the vasculature and superficial tissue structures of the mucosa [9]. In NBI mode, hemoglobin absorbs light through the tissue surface, thereby increasing the visibility of the capillary and surface structure. NBI improves tumor visibility by enhancing the contrast between vascularized lesions and normal mucosal [10-12]. These features allow NBI to detect small or flat early tumors that are difficult to observe by WLI [13]. Systems integrating both NBI and WLI are already available. Upon selection, a narrowband filter is inserted in front of the white light source to activate the NBI mode [14, 15].

The majority of NMIBC recurrences after transurethral resection (TUR), particularly the intermediate and high levels of T1, are overlooked and residual cancers $[16,17]$. The residual tumor rate after the first electro-TUR is approximately $33.8-36 \%[18,19]$. Among cases of NMIBC, $17.1 \%$ tumors were detected by NBI only, whereas $1.9 \%$ tumors were found by WLI only [20]. Additionally, $42 \%$ patients with positive urine cytology and negative WLI cystoscopy presented with BCs when undergoing NBI cystoscopy [21]. NBI cystoscopy facilitates the early detection and diagnosis of small lesions, reducing the rate of residual tumors.

Narrow band imaging-assisted transurethral resection (NBI-TUR) decreased the residual tumor rate significantly compared with white light imaging-assisted transurethral resection (WLI-TUR) in a matched cohort. The residual tumor rates at first follow-up for WLI-TUR and NBI-TUR were $30.5 \%$ and $15.0 \%$, respectively [22]. Angelo found that NBI-UTR reduced the recurrence risk of NMIBC by at least $10 \%$ at $1 \mathrm{yr}$ [23]. However, the study sample came from a single center and was limited, and we require many multi-center clinical trials for further verification. Thus, we aim to perform a systematic review and meta-analysis of randomized clinical trials (RCTs) that have evaluated the efficacy of NBI-TUR for NMIBC to provide the basis for clinical decision-making.

\section{EVIDENCE ACQUISITION}

\section{Identification of eligible trials}

The protocol was registered in the Prospective Register of Systematic Reviews (PROSPERO number CRD 42016038895). The study was designed and performed according to the Preferred Reported Items for Systematic Reviews and Meta-analysis guidelines [24]. Study designs considered for inclusion were random controlled trials and retrospective cohort studies assessing NBI-TUR for NMIBC.

The inclusion criteria were as follows: Participants: patients with NMIBC. Interventions: WLI-TUR and NBITUR as treatment modalities. Comparisons: WLI-TUR vs. NBI-TUR. Outcomes: recurrence risk.

The exclusion criteria were as follows: muscle invasive $\mathrm{BC}$, non-comparative studies, no comparison between WLI-TUR and NBI-TUR, no detailed data regarding the recurrence risk in the WLI-TUR or NBITUR group.

The search was performed using PubMed, Medline, Ovid, Embase, Cochrane Library and Web of Science. We used the following search terms in all databases: ("narrow band imaging" or "NBI") AND ("bladder cancer" or "bladder tumor" or "bladder carcinoma") AND ("transurethral resection"). The references in the included articles were further examined to identify additional qualified clinical trials. Trials enrolling patients with NBITUR or WLI-TUR were included. When the same trial was reported more than once, the most recent information was considered in the analysis. 


\section{Data collection}

The following data were collected from each eligible trial, if available:

Main information: age, sex, clinical status, number of tumors, tumor grade, tumor stage, and tumor size.

Details of study treatment: modality of cystoscopy before TUR, type of TUR, modality of cystoscopy in TUR, adjuvant topical therapy after TUR.

Study design: primary end point, second end point, study type.
Patient enrollment and follow-up: start and end dates of the study; number of patients assigned to the experimental arm, number of patients assigned to the control arm, follow-up.

Recurrence risk: total number of patients, number of recurrences in each arm, $p$ value.

The randomization quality was evaluated in each study based on the information available in the publication $[23,25-29]$.

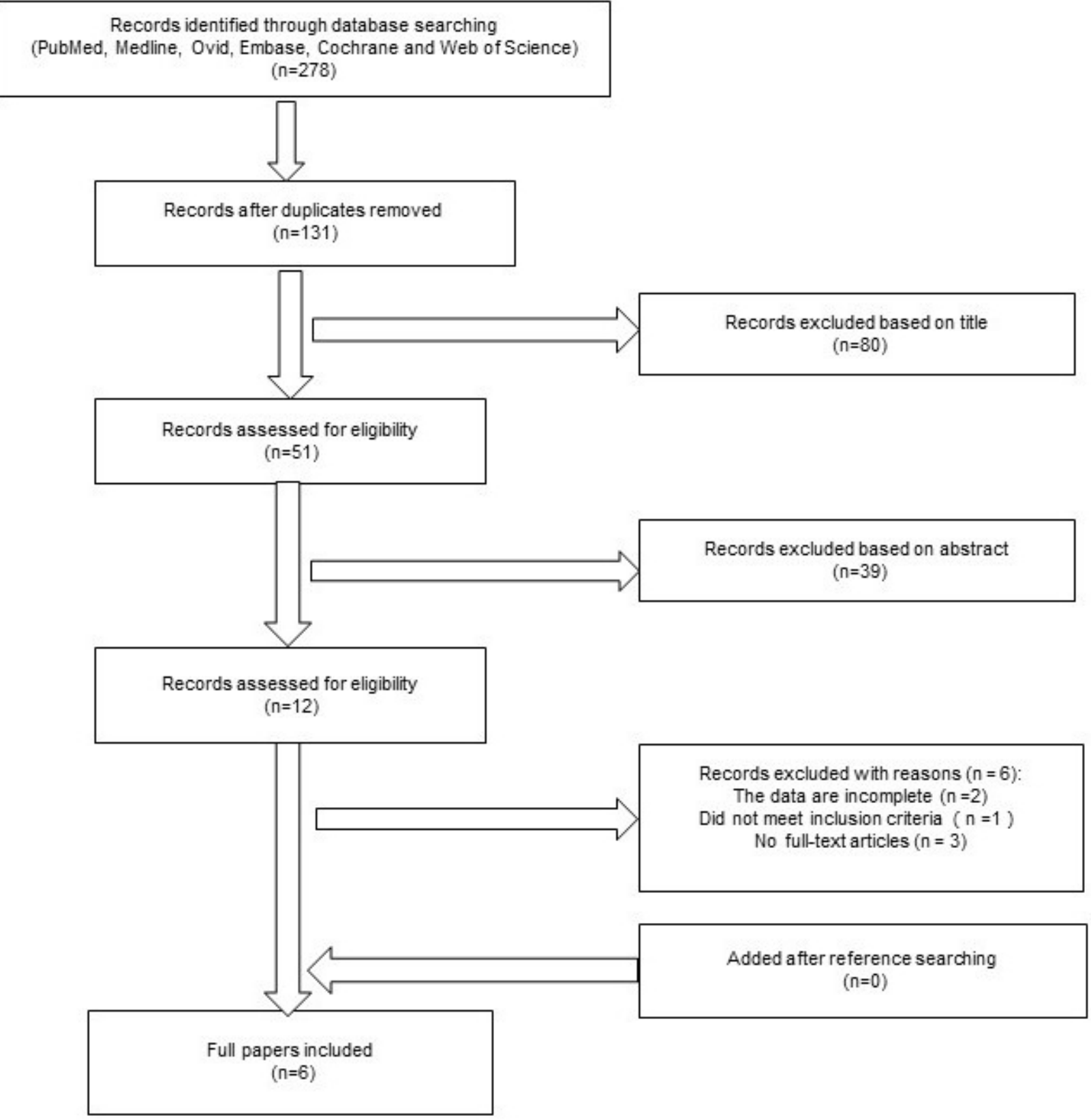

Figure 1: Preferred reporting items for the systemic review and meta-analysis flowchart. Six papers were ultimately included. 


\section{Statistical methods}

After data extraction, the data were analyzed using Review Manager software (RevMan v.5.3; The Nordic Cochrane Center, Copenhagen, Denmark). Risk ratios (RRs) were used for dichotomous variables, with $95 \%$ confidence intervals (CIs). We evaluated the methodological heterogeneity among the selected literature, and we used the $\chi 2$ test and I2 scores to measure statistical heterogeneity. If the result was p $>0.1$ and I $<$ $50 \%$, we considered the heterogeneity low. We used a fixed-effects model to assess the data, and the p-value for significance was set at $<0.05$.

\section{EVIDENCE SYNTHESIS}

\section{Characteristics and quality of the trials}

Supplementary Figure 1 shows the trial selection process for the search performed in February of 2016. Of 278 published papers, 272 were excluded, and 6 papers

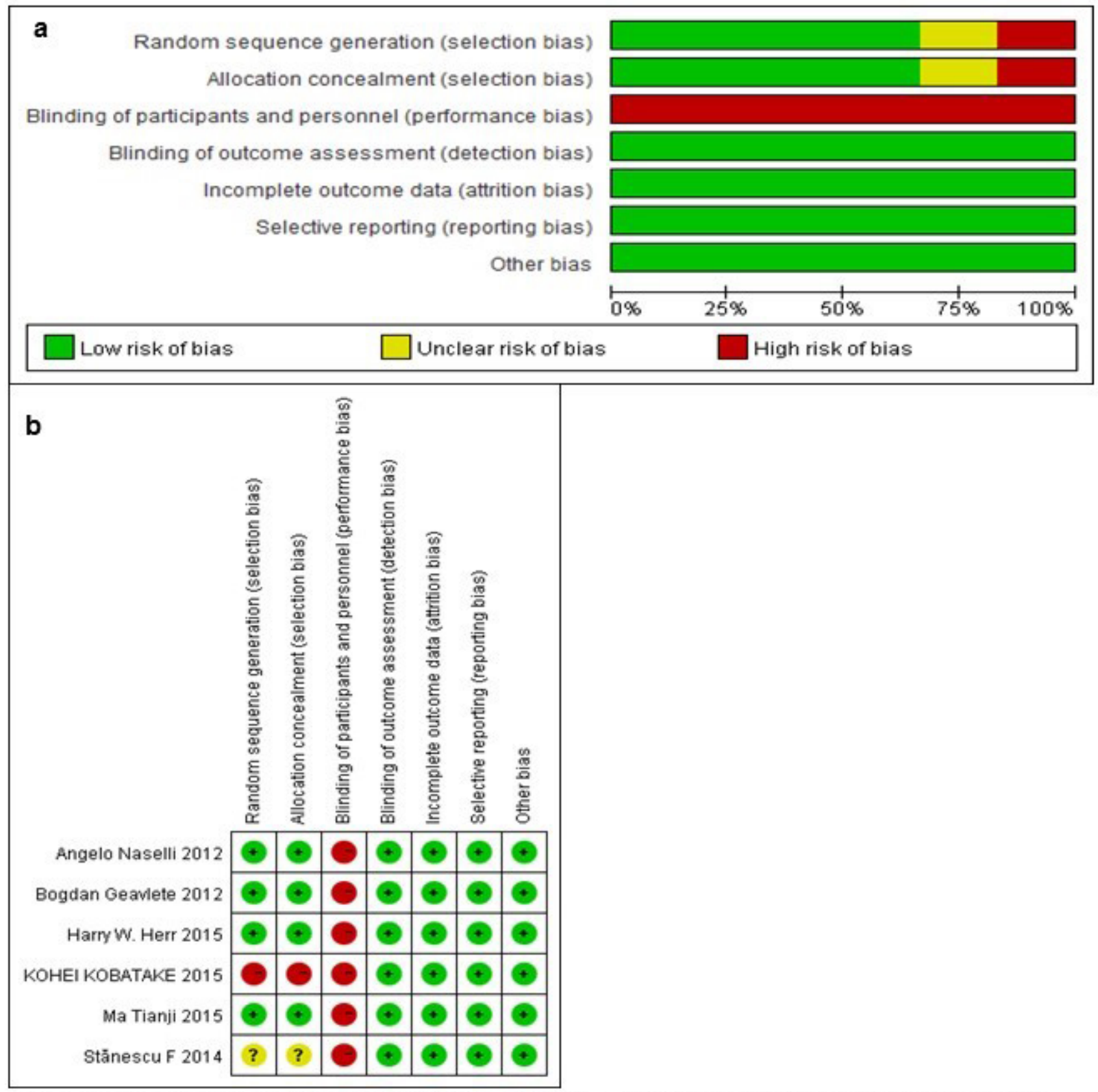

Figure 2: Risk of bias graph (a) and summary (b). Review author's judgments for each risk of bias item for each included study. Green: low risk of bias, red: high risk of bias and yellow: unclear risk of bias. 


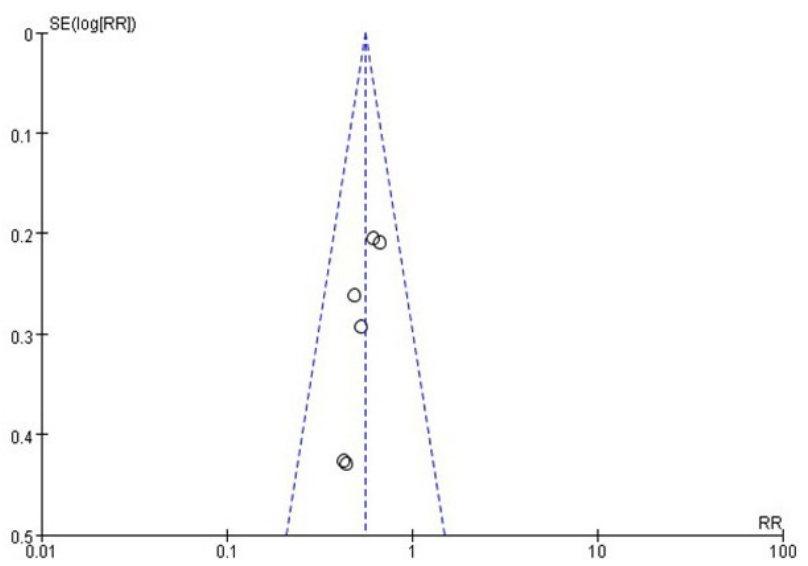

Figure 3: Funnel plot on recurrence risk. Little evidence of publication bias was demonstrated of the funnel plots.

a. The 3-mo recurrence risk

NBI-TUR

WLI-TUR

Risk Ratio

Risk Ratio

\begin{tabular}{lrrrrrr} 
Study or Subaroup & Events & Total & Events & Total & Weight & M-H, Fixed, 95\% Cl \\
\hline Angelo Naselli 2012 & 3 & 76 & 12 & 72 & $18.3 \%$ & $0.24[0.07,0.80]$ \\
Bogdan Geavlete 2012 & 2 & 89 & 6 & 90 & $8.9 \%$ & $0.34[0.07,1.63]$ \\
Harry W. Herr 2015 & 14 & 127 & 30 & 127 & $44.6 \%$ & $0.47[0.26,0.84]$ \\
KOHEI KOBATAKE 2015 & 2 & 57 & 3 & 78 & $3.8 \%$ & $0.91[0.16,5.28]$ \\
Ma Tianji 2015 & 5 & 86 & 17 & 92 & $24.4 \%$ & $0.31[0.12,0.82]$ \\
& & & & & & \\
Total (95\% Cl) & 26 & 435 & & 459 & $100.0 \%$ & $0.39[0.26,0.60]$ \\
Total events & 268 & & &
\end{tabular}

Heterogeneity. $\mathrm{Chi}^{2}=2.12, \mathrm{df}=4(\mathrm{P}=0.71) ; \mathrm{P}^{2}=0 \%$

Test for overall effect $Z=4.30(P<0.0001)$

M.H. Fixed. $95 \% \mathrm{Cl}$

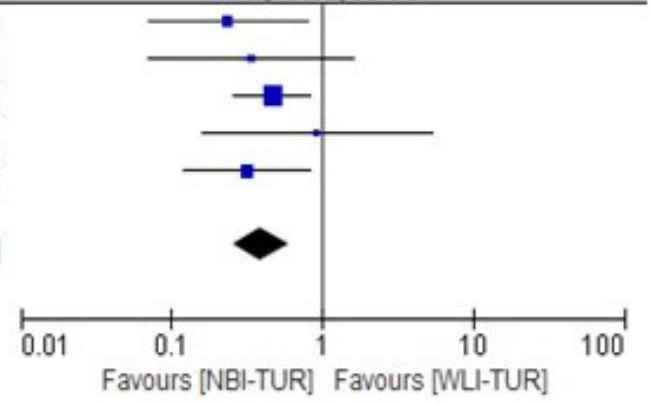

\section{b. The 1-yr recurrence risk}

NBI-TUR

WLI-TUR

Risk Ratio

Risk Ratio

Study or Subqroup Events Total Events Total Weight M-H, Fixed, 95\% Cl

Angelo Naselli 2012

$\begin{array}{llllll}24 & 76 & 37 & 72 & 29.1 \% & 0.61[0.41,0.92]\end{array}$

Bogdan Geavlete 2012

$\begin{array}{lllll}7 & 89 & 16 & 90 & 12.2 \%\end{array}$

$\begin{array}{ll}12 & 57 \\ 16 & 86\end{array}$

$31-78 \quad 20.1 \%$

$0.44[0.19,1.02]$

KOHEI KOBATAKE 2015

$35-78 \quad 20.1 \%$

$0.53[0.30,0.94]$

Ma Tianji 2015

Stănescu F 2014

793

$17 \quad 97 \quad 12.7 \%$

$.49[0.29,0.82]$

$0.43[0.19,0.99]$

Total $(95 \% \mathrm{Cl})$

401

Total events

$429100.0 \% \quad 0.52[0.40,0.67]$

Heterogeneity: $\mathrm{Ch}^{2}=1.07, \mathrm{df}=4(\mathrm{P}=0.90) ; \mathrm{F}^{2}=0 \%$

Test for overall effect $Z=5.05$ ( $P<0.00001)$

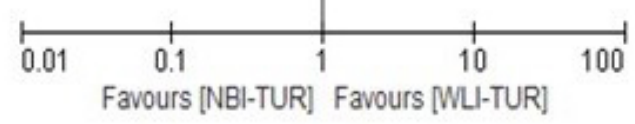

c. The 2-yr recurrence risk

NBI-TUR

WLI-TUR

Risk Ratio

Risk Ratio

Study or Subaroup Events Total Events Total Weight M-H. Fixed, 95\% Cl

M.H. Fixed, $95 \% \mathrm{Cl}$

\begin{tabular}{|c|c|c|c|c|c|c|}
\hline Harry W. Herr 2015 & 28 & 127 & 42 & 127 & $64.1 \%$ & $0.67[0.44,1.00]$ \\
\hline Stănescu F 2014 & 11 & 93 & 24 & 97 & $35.9 \%$ & $0.48[0.25,0.92]$ \\
\hline Total $(95 \%$ Cl) & & 220 & & 224 & $100.0 \%$ & $0.60[0.42,0$ \\
\hline Total events & 39 & & 66 & & & \\
\hline \multicolumn{7}{|c|}{ Heterogeneity. $C h i^{2}=0.72, d f=1(P=0.40) ; 1^{2}=0 \%$} \\
\hline
\end{tabular}

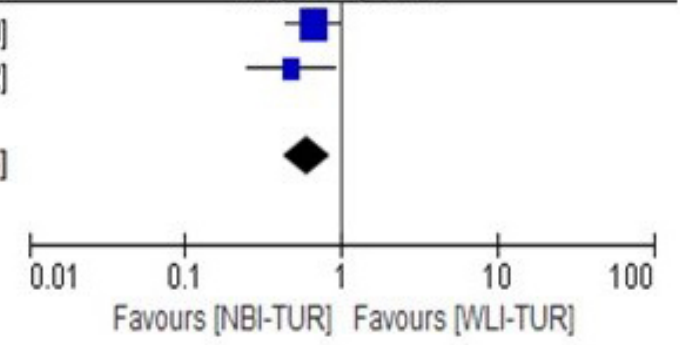

Figure 4: Pairwise meta-analysis for the recurrence risk of NMIBC during the follow-up period. NBI-TUR was associated with a significant benefit in the 3-mo recurrence risk a., 1-yr recurrence risk b. and 2-yr recurrence risk c. 
Table 1: Baseline characteristics of the included trials

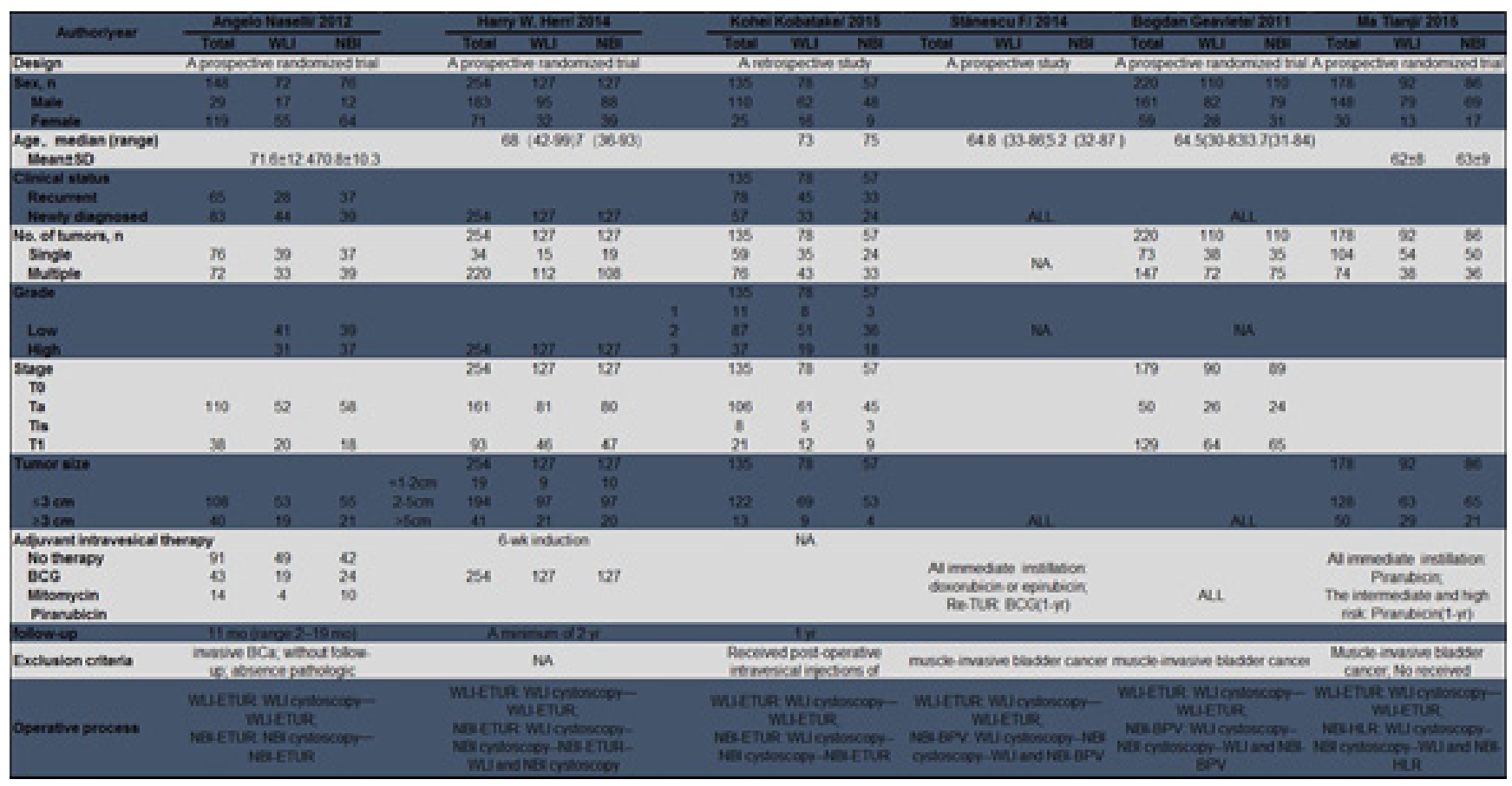

were eligible for inclusion [23, 25-29]. The patients with NMIBC in each trial were randomized into two arms using methods such as random permuted blocks and sealed envelopes.

The main analysis was performed considering the six comparisons of NBI-TUR vs. WLI-TUR. Three trials performed narrow band imaging-assisted electrotransurethral resection (NBI-ETUR) and WLI-assisted electro-TUR (WLI-ETUR) [23, 26, 27]. Two trials performed narrow band imaging-associated bipolar plasma vaporization (NBI-BPV) and WLI-ETUR [25, 29]. In those two trials, NMIBC was $>3 \mathrm{~cm}$ in diameter in all patients. The last trial performed narrow band imaging-assisted holmium laser resection (NBI-HLR) and WLI-ETUR [28]. A holmium laser has high efficiency and safety features and provides a new method for the treatment of BC.

For the recurrence risk, a subgroup analysis was performed in patients according to the type of TUR, which included NBI-ETUR, NBI-BPV and NBI-HLR.

Figure 2 shows the quality assessment regarding the six available trials, as measured by the Cochrane Collaboration's tool for assessing the risk of bias. In all trials, patients assigned to the experimental arm received NBI-TUR, and the control arm received WLI-TUR. Five trials included in our analysis were RCTs, and one trial was a retrospective cohort that had a high risk of bias compared with the RCTs. One trial did not introduce the random sequence generation. All of the trials failed to blind the researchers to the treatment, which may affect the final results. Five trials exhibited a low risk of bias, and one study exhibited a high risk of bias.
Regarding the $\mathrm{BC}$ recurrence risk, little evidence of publication bias was observed on visual or statistical examinations of the funnel plot (Figure 3 ).

\section{Patient characteristics}

Overall, 1084 patients were included in the six trials in the meta-analysis; $556(51.3 \%)$ were assigned to WLI-ETUR, and 528 (48.7\%) were assigned to NBITUR, including 260 (24.0\%) assigned to NBI-ETUR, 182 $(16.8 \%)$ to NBI-BPV and $86(8.0 \%)$ to NBI-HLR. The main characteristics of the 1084 patients are described in Table 1.

\section{Recurrence risk}

Overall, cancer recurred in 283 patients in the two arms during the follow-up period, as shown in Table 2. As shown in Figure 4, NBI-TUR in NMIBC was associated with a significant benefit in the 3-mo (RR: 0.39 ; 95\% CI, 0.26-0.60; $\mathrm{p}<0.0001$ ), 1-yr (RR: 0.52; 95\% CI, 0.40-0.67; $\mathrm{p}<0.00001)$ and 2-yr (RR: 0.60; 95\% CI, 0.42-0.85; $\mathrm{p}=$ $0.004)$ recurrence risks compared with WLI-TUR. There was no evidence of significant heterogeneity among the trials $(\mathrm{p}=0.71 ; \mathrm{I} 2=0 \%),(\mathrm{p}=0.90 ; \mathrm{I} 2=0 \%)$ and $(\mathrm{p}=$ $0.40 ; \mathrm{I} 2=0 \%$, respectively.

A subgroup analysis was performed for all patients with operation methods of the experimental group enrolled in the six trials (Figure 5). Subgroup analyses did not show that different surgical methods for recurrence of NMIBC 
Table 2: The recurrence risk of NMIBC during the follow-up period.

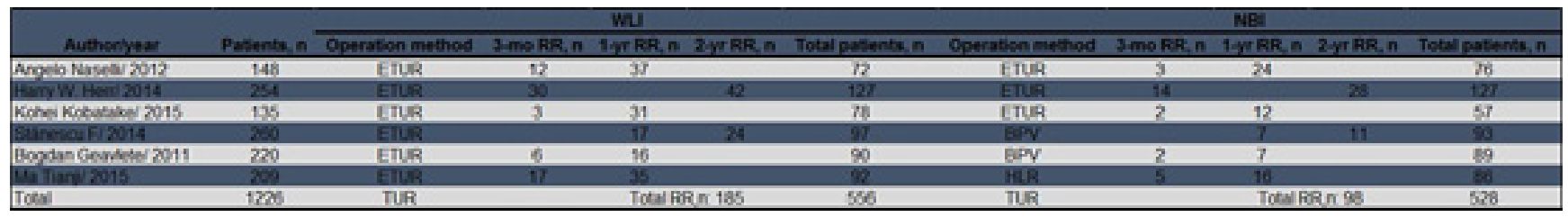

had a significant interaction $(p=0.53)$. The $R R$ for the operation method was 0.61 (95\% CI, 0.47-0.79) in patients with NBI-ETUR, 0.46 (95\% CI, 0.28-0.78) in patients with NBI-BPV and 0.49 (95\% CI, 0.29-0.82) in patients with NBI-HLR.

\section{DISCUSSION}

This meta-analysis shows that NBI-TUR for NMIBC has a significant improvement in recurrence risk compared with WLI-TUR.

A quantitative synthesis of the currently available evidence regarding this operation method could be very helpful for clinical decisions because these six trials showed a significant benefit associated with NBI-TUR for the treatment of NMIBC. To the best of our knowledge, no other trials have reported the recurrence risk of NBITUR in NMIBC, and this meta-analysis synthesizes all the evidence produced to date. Although all the trials showed the benefit of NBI-TUR for NMIBC in terms of the recurrence risk, the study sample was a single-center study and was limited, and we require many multi-center clinical trials to further verify the findings. Therefore, this meta-analysis synthesizes six trials to test the efficacy of NBI-TUR in NMIBC.

Approximately $70 \%$ of cases of NMIBC present with pTa, $20 \%$ with pT1, and $10 \%$ with CIS lesions [1]. Many studies have reported routine detection of residual

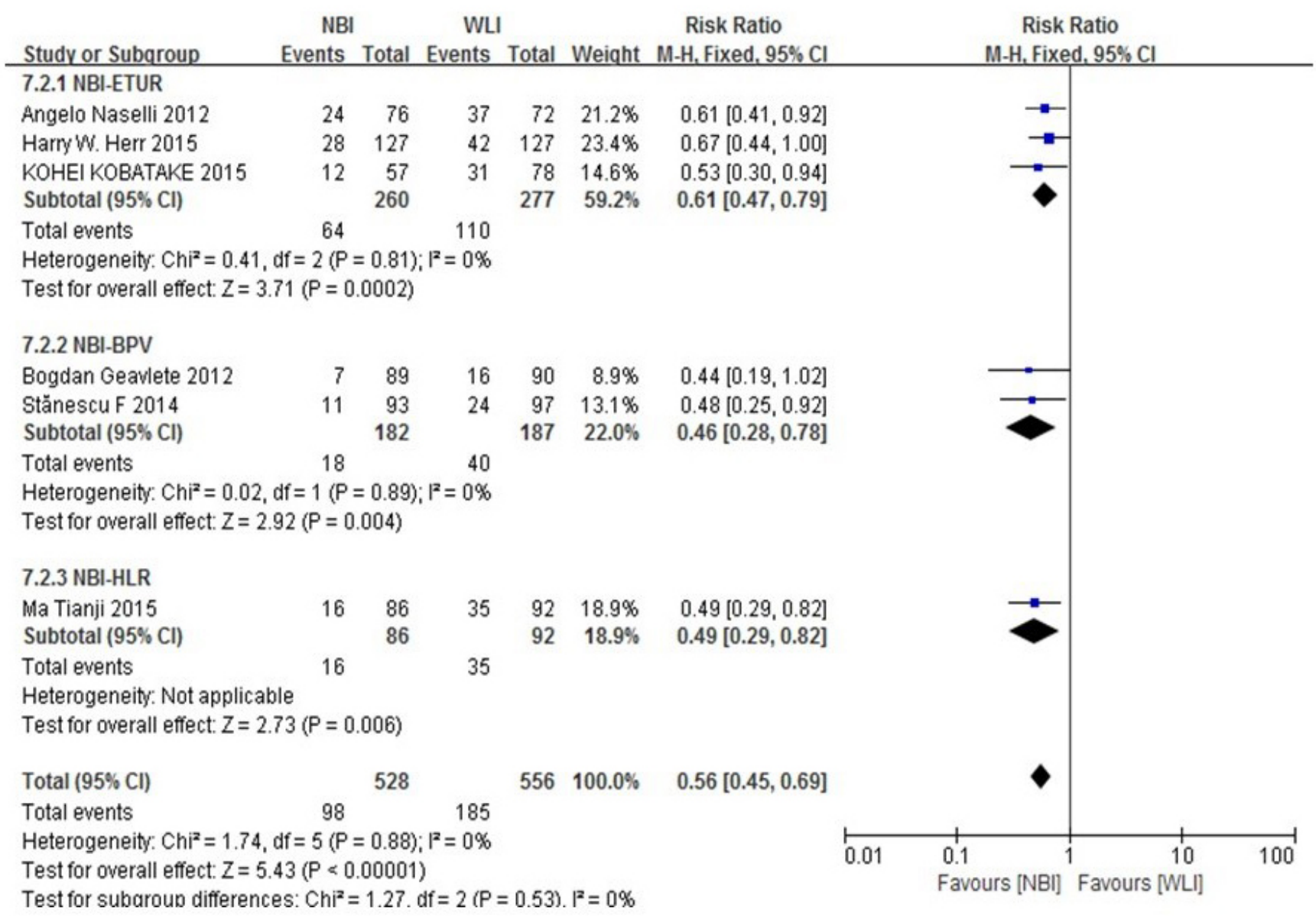

Figure 5: Subgroup analysis for operation method. Subgroup analyses did not show that different surgical methods for recurrence of NMIBC had a significant interaction. 
tumors during second TUR [30]. For example, Babjuk et al. [31] reported tumors in $32-36 \%$ of patients at a second TUR within 7 weeks after the initial TUR, particularly in patients with TaG3 disease or multiple tumors [31, 32]. Residual transitional cell tumors were observed in $44 \%$ of patients after re-resection of the original tumor site [16]. For high-grade tumors, this rate could be as high as $70 \%$ [33]. The probabilities of recurrence after TUR at $1 \mathrm{yr}$ ranged from $15 \%$ to $61 \%$, and the probabilities of recurrence ranged from $31 \%$ to $78 \%$ at $5 \mathrm{yr}$ [7]. Sylvester et al. reported that the median time to first recurrence was $2.7 \mathrm{yr}$ [7]. The high rate of recurrence is a major challenge when treating NMIBC. Brausi et al. showed that overlooked tumors during the initial TUR, not true relapse, are responsible for recurrence. The rate of 'recurrences' was $3.4-20.6 \%$ for single tumors and $7.4-45.6 \%$ for multiple tumors [6]. Therefore, some researchers have suggested that recurrence can be divided into true recurrence and residual tumors that were likely overlooked on the initial TUR. Improving the detection rate of NMIBC will help reduce recurrences.

To date, the standard operation is WLI-TUR, but this operation might miss small papillary tumors or CIS. The detection rate of NBI was $94.7 \%$ vs. $79.2 \%$ for WLI ( $p<$ .001). NBI cystoscopy as optical enhancement technology improves the detection of primary and recurrent NMIBC over WLI [34]. Although some trials have proven the advantage of using NBI-TUR, for example Herr [26] reported an $11 \%$ reduction in the recurrence rate using NBI-TUR and Naselli et al. [23] showed that NBI-TUR reduces the 1-yr recurrence risk of NMIBC by at least $10 \%$, these trials were single-center studies and were limited [23, 26].

Our systematic review could be regarded as a multi-center, large sample study, but it also has some limitations. First, not all experiments were prospective randomized controlled trials. The study by Kobatake et al. [27] was a retrospective cohort study. Major biases with retrospective cohort studies could impact the recall of former exposure to risk variables, including selection bias and information bias. It could be very difficult to make accurate comparisons between the NBI-TUR and WLITUR arms. Second, in five trials, the operative process was unreasonable, and NBI cystoscopy was regarded as a second procedure to inspect and resect visible or suspected tumors. This process would result in observer bias because NBI would be used as a supplement to conventional WLI and may prove to be better than either modality used alone. Furthermore, operators may break blindness and take more time in NBI-TUR than in WLITUR. Third, the design of the experimental group was not perfect. In the control group, all patients with NMIBC in six trials underwent WLI-ETUR, whereas in the experimental group, patients with NMIBC in three trials underwent NBI-ETUR, patients in two trials underwent NBI-BPV and patients in one trial underwent NBI-HLR.
The different operation methods between the experimental group and control group would affect the recurrence risk of NMIBC. Fourth, cost and time implication analyses for NMIBC in the NBI-TUR were not performed. Fifth, intensive analyses of different sub-groups based on the clinical characteristics of patients are needed to compare recurrence rates. Gender, age, tumor stage, grade and adjuvant topical therapy should also be considered in future analyses. These factors are associated with the recurrence risk, but the lack of complete data limited our ability to analyze these factors. Finally, long-term recurrence risk and overall survival analyses are needed.

Despite the above limitations, we still believe our review has clinical significance. All trials showed that NBI-TUR reduced the recurrence risk of NMIBC compared with WLI-TUR, in particular Ma et al. reported the 1 -yr recurrence risk was $18.60 \%$ in the NBI-HLR and $38.04 \%$ in the WLI-ETUR [28]. NBI-HLR reduced the 1 -yr recurrence risk significantly by $19.44 \%$. Geavlete et al. reported a slight difference of $9.9 \%$ in the $1-y r$ recurrence risk for $\mathrm{NMIBC}>3 \mathrm{~cm}$, with rates of $7.9 \%$ for NBI-BPV vs. $17.8 \%$ for WLI-ETUR [25]; however, the recurrence risk was higher than that reported by Ma et al. [28]. In another study, the 1-yr recurrence risk after NBI-BPV compared with WLI-ETUR was $7.2 \%$ vs. $18.3 \%$, respectively. That study also reported that the $2-y r$ recurrence risk after NBI-BPV was $11.5 \%$ vs. $25.8 \%$ for WLI-ETUR [29]. Herr reported that the 2-yr recurrence risk was $22 \%$ vs. $33 \%$, representing a reduction of $11 \%$ for NBI-TUR, and the 2-yr recurrence-free survival and mean survival time were 22 mo and 19 mo, respectively [26]. Kobatake et al. reported that the recurrence probability increased in the NBI group from $3.5 \%$ at 3 mo to $21.1 \%$ at $1 \mathrm{yr}$ and increased from $3.8 \%$ to $39.7 \%$ in the control group [27]. The last trials showed that the recurrence rates increased from $4 \%$ at 3 mo to $32 \%$ at 1 yr for NBITUR and from $12 \%$ to $51 \%$ for WLI-TUR. Because of the increasing trend of tumor recurrence, some recurrence is attributed to causes other than missed tumors, such as the field cancerization effect [35] or the clonal origin of urothelial cancer [13], which are not yet completely understood. The recurrence mechanism of NMIBC should be further studied.

One aspect to consider is whether we are ready to change practices based on this review. First, the above trials showed that NBI-TUR can reduce the recurrence risk of NMIBC compared with WLI-TUR at 3 mo, $1 \mathrm{yr}$ and 2 yr. Photodynamic diagnosis (PDD) is another new imaging technology that has been recently introduced. PDD requires the perfusion of photoactive porphyrin precursors, such as 5-aminolevulinic acid (5-ALA) or hexylaminolevulinate (HAL), and could improve the detection rate of BC. Fluorescence cystoscopy improves the detection rate of papillary tumors [36] and CIS [37] compared with WLI cystoscopy. In a randomized prospective study of treatment outcomes, the tumor-free 
recurrence rate at 1 -yr after TUR using 5-ALA was $18 \%$ lower than that after WLI-TUR [38]. A review reported that the recurrence rate among resected patients was not significantly different between either 5-ALA or HAL and NBI-TUR [39]. Second, recurrence increases the workload and the diagnosis, treatment and, in particular, follow-up of NMBIC, thus increasing costs [32]. Botteman et al. [40] calculated that $\mathrm{BC}$ is currently the most expensive tumor because existing diagnosis, treatment and followup methods are not cost-effective. Avritscher et al. [41] calculated comparable costs and found that the treatment of recurrences accounts for approximately $60 \%$ of these costs. Third, PDD requires perfusion of photosensitizers, whereas NBI cystoscopy does not require additional invasive procedures [42]. NBI-TUR is superior to PDD because the specificity of PDD for the diagnosis of $\mathrm{BC}$ is significantly decreased in the patients who were treated with bladder instillation [43]. In addition to the drawback of photodynamic diagnostics, the relatively low specificity of PDD must be considered [44]. We believe that NBITUR should be further studied and generalized.

However, NBI cystoscopy has limitations. First, because NBI has no tumor specificity and can only provide morphological features of suspicious lesions, this increased sensitivity and decreased specificity will eventually lead to an increased rate of false-positives [45]. Second, bladder instillation to detect lesion recurrence due to the lack of NBI examination standards may lead to unnecessary biopsy [46]. Third, the light emitted from abnormal lesions is strongly absorbed by hemoglobin, and thus, abnormal conditions such as bladder bleeding or active inflammation make NBI difficult to diagnose [47].

This review will aid the more precise identification of indications for the application of NBI in NMIBC.

\section{CONCLUSIONS}

Our meta-analysis clearly shows NBI-TUR can significantly reduce the recurrence risk of NMIBC compared with WLI-TUR at $3 \mathrm{mo}, 1 \mathrm{yr}$ and $2 \mathrm{yr}$. Considering the absence of heterogeneity among the six trials, the result is believable. If our findings are confirmed by large multi-center validating studies, NBI-TUR would be used in the clinic.

\section{Abbreviations}

WLI: white light imaging; NBI: narrow band imaging; TUR: transurethral resection; $\mathrm{BC}$ : bladder cancer; NMIBC: non-muscle invasive bladder cancer; NBI-TUR: narrow band imaging-assisted transurethral resection; WLI-TUR: white light imaging-assisted transurethral resection; WLI-ETUR: white light imagingassisted electro-transurethral resection; NBI-ETUR: narrow band imaging-assisted electro-transurethral resection; NBI-BPV: narrow band imaging-associated bipolar plasma vaporization; NBI-HLR: narrow band imaging-assisted holmium laser resection; RCT: randomized clinical trial; $\mathrm{CI}$ : confidence interval; RR: risk ratio; mo: month; yr: year; CIS: carcinoma in situ; PDD: photodynamic diagnosis; 5-ALA: 5-aminolevulinic acid; HAL: hexylaminolevulinate.

\section{CONFLICTS OF INTEREST}

The authors report no declarations of interest.

\section{FUNDING}

This study was funded by the National Natural Science Foundation of China (grant No. 81202016), a 59-class General Financial Grant from the China Postdoctoral Science Foundation (grant No. 2016M590638), the National Natural Science Foundation of China (grant No. 81300629 and grant No. 81572534), and the Key Science and Technology Program of Shandong Province, China (grant No. 2015GSF118096).

\section{Author contributions}

WK designed the study, performed the literature search and selection, data extraction, quality assessment, and statistical analysis, and drafted the manuscript; ZC performed the literature selection, statistical analysis, and quality assessment; QC performed the literature search and data extraction; XJ, DZ and $\mathrm{HZ}$ provided critical comments and revised the manuscript. All authors have made an intellectual contribution to the manuscript and approved the submission.

\section{REFERENCES}

1. Kirkali Z, Chan T, Manoharan M, Algaba F, Busch C, Cheng L, Kiemeney L, Kriegmair M, Montironi R, Murphy WM. Bladder cancer: epidemiology, staging and grading, and diagnosis. Urology. 2005; 66: 4-34.

2. Ferlay J, Shin H, Bray F, Forman D, Mathers C, Parkin D. GLOBOCAN 2008, Cancer Incidence and Mortality Worldwide: IARC CancerBase No. 10. 2010 Lyon, France: International Agency for Research on Cancer; 2013. Available from: globocan.iarc.fr.

3. Goh AC, Lerner SP. Application of new technology in bladder cancer diagnosis and treatment. World J Urol. 2009; 27: 301-307.

4. Herr HW. Natural history of superficial bladder tumors: 10- to 20-year follow-up of treated patients. World J Urol. 1997; 15: 84-88.

5. Holmang S, Hedelin H, Anderstrom C, Holmberg E, Busch $\mathrm{C}$, Johansson SL. Recurrence and progression in low grade 
papillary urothelial tumors. J Urol. 1999; 162: 702-707.

6. Brausi M, Collette L, Kurth K, van der Meijden AP, Oosterlinck W, Witjes JA, Newling D, Bouffioux C, Sylvester RJ. Variability in the recurrence rate at first follow-up cystoscopy after TUR in stage Ta T1 transitional cell carcinoma of the bladder: a combined analysis of seven EORTC studies. Eur Urol. 2002; 41: 523-531.

7. Sylvester RJ, van der Meijden APM, Oosterlinck W, Witjes JA, Bouffioux C, Denis L, Newling DWW, Kurth K. Predicting recurrence and progression in individual patients with stage Ta T1 bladder cancer using EORTC risk tables: a combined analysis of 2596 patients from seven EORTC trials. Eur Urol. 2006; 49: 466-477.

8. Millán-Rodríguez F, Chéchile-Toniolo G, Salvador-Bayarri J, Palou J, Vicente-Rodríguez J. Multivariate analysis of the prognostic factors of primary superficial bladder cancer. J Urol. 2000; 163: 73-78.

9. Herr HW, Donat SM. A comparison of white-light cystoscopy and narrow-band imaging cystoscopy to detect bladder tumour recurrences. BJU Int. 2008; 102: 11111114.

10. Liu JJ, Droller MJ, Liao JC. New optical imaging technologies for bladder cancer: considerations and perspectives. J Urol. 2012; 188: 361-368.

11. Cauberg ECC, Kloen S, Visser M, De La Rosette JJMCH, Babjuk M, Soukup V, Pesl M, Duskova J, De Reijke TM. Narrow band imaging cystoscopy improves the detection of nonmuscle-invasive bladder cancer. Urology. 2010; 76: 658-663.

12. Herr HH. Narrow band imaging cystoscopy. Urol Oncol. 2011; 29: 353-357.

13. Hirata I, Nakagawa Y, Ohkubo M, Yahagi N, Yao K. Usefulness of magnifying narrow-band imaging endoscopy for the diagnosis of gastric and colorectal lesions. Digestion. 2012; 85: 74-79.

14. Kuznetsov K, Lambert R, Rey JF. Narrow-band imaging: potential and limitations. Endoscopy. 2006; 38: 76-81.

15. Wong Kee Song LM, Adler DG, Conway JD, Diehl DL, Farraye FA, Kantsevoy SV, Kwon R, Mamula P, Rodriguez B, Shah RJ, Tierney WM. Narrow band imaging and multiband imaging. Gastrointest Endosc. 2008; 67: 581589.

16. Klän R, Loy V, Huland H. Residual tumor discovered in routine second transurethral resection in patients with stage T1 transitional cell carcinoma of the bladder. J Urol. 1991; 146: 316-318.

17. Herr HW. The value of a second transurethral resection in evaluating patients with bladder tumors. J Urol. 1999; 162: 74-76.

18. Kolozsy Z. Histopathological "self control" in transurethral resection of bladder tumours. Br J Urol. 1991; 67: 162-164.

19. Divrik RT, Yildirim Üt, Zorlu F, Özen H. The effect of repeat transurethral resection on recurrence and progression rates in patients with $\mathrm{T} 1$ tumors of the bladder who received intravesical mitomycin: a prospective, randomized clinical trial. J Urol. 2006; 175: 1641-1644.

20. Shen Y-J, Zhu Y-P, Ye D-W, Yao X-D, Zhang S-L, Dai B, Zhang H-L, Zhu Y. Narrow-band imaging flexible cystoscopy in the detection of primary non-muscle invasive bladder cancer: a "second look" matters? Int Urol Nephrol. 2012; 44: 451-457.

21. Zhu Y-P, Shen Y-J, Ye D-W, Wang C-F, Yao X-D, Zhang S-L, Dai B, Zhang H-L, Shi G-H. Narrow-band imaging flexible cystoscopy in the detection of clinically unconfirmed positive urine cytology. Urol Int. 2011; 88: 8487.

22. Cauberg ECC, Mamoulakis C, de la Rosette JJMCH, de Reijke TM. Narrow band imaging-assisted transurethral resection for non-muscle invasive bladder cancer significantly reduces residual tumour rate. World J Urol. 2011; 29: 503-509.

23. Naselli A, Introini C, Timossi L, Spina B, Fontana V, Pezzi R, Germinale F, Bertolotto F, Puppo P. A randomized prospective trial to assess the impact of transurethral resection in narrow band imaging modality on non-muscleinvasive bladder cancer recurrence. Eur Urol. 2012; 61: 908-913.

24. Moher D, Liberati A, Tetzlaff J, Altman DG. Preferred reporting items for systematic reviews and meta-analyses: the PRISMA statement. Ann Intern Med. 2009; 151: 264269.

25. Geavlete B, Multescu R, Georgescu D, Stanescu F, Jecu M, Geavlete P. Narrow band imaging cystoscopy and bipolar plasma vaporization for large nonmuscle-invasive bladder tumors - results of a prospective, randomized comparison to the standard approach. Urology. 2012; 79: 846-851.

26. Herr HW. Randomized trial of narrow-band versus whitelight cystoscopy for restaging (second-look) transurethral resection of bladder tumors. Eur Urol. 2015; 67: 605-608.

27. Kobatake K, Mita K, Ohara S, Kato M. Advantage of transurethral resection with narrow band imaging for nonmuscle invasive bladder cancer. Oncol Lett. 2015; 10: 1097-1102.

28. Ma T, Wang W, Jiang Z, Shao G, Guo L, Li J, Zhang L, Liu $Y$. Narrow band imaging-assisted holmium laser resection reduced the recurrence rate of non-muscle invasive bladder cancer: a prospective, randomized controlled study. National Medical Journal of China. 2015; 95: 3032-3035.

29. Stanescu F, Geavlete B, Georgescu D, Jecu M, Moldoveanu C, Adou L, Bulai C, Ene C, Geavlete P. NBI - plasma vaporization hybrid approach in bladder cancer endoscopic management. J Med Life. 2014; 7: 155-159.

30. Jakse G, Algaba F, Malmström P-U, Oosterlinck W. A second-look TUR in T1 transitional cell carcinoma: why? Eur Urol. 2004; 45: 539-546.

31. Babjuk M, Soukup V, Petrík R, Jirsa M, Dvorácek J. 5-aminolaevulinic acid-induced fluorescence cystoscopy during transurethral resection reduces the risk of recurrence 
in stage Ta/T1 bladder cancer. BJU Int. 2005; 96: 798-802.

32. Lee CSD, Yoon CY, Witjes JA. The past, present and future of cystoscopy: the fusion of cystoscopy and novel imaging technology. BJU Int. 2008; 102: 1228-1233.

33. Al'-Shukri S, Danil'chenko DI, Kënig F, Shnorr D. [ALA fluorescent diagnosis of bladder cancer]. Urologiia. 2000. 48-50.

34. Cauberg ECC, Kloen S, Visser M, de la Rosette JJMCH, Babjuk M, Soukup V, Pesl M, Duskova J, de Reijke TM. Narrow band imaging cystoscopy improves the detection of non-muscle-invasive bladder cancer. Urology. 2010; 76: 658-663.

35. Hafner C, Knuechel R, Zanardo L, Dietmaier W, Blaszyk H, Cheville J, Hofstaedter F, Hartmann A. Evidence for oligoclonality and tumor spread by intraluminal seeding in multifocal urothelial carcinomas of the upper and lower urinary tract. Oncogene. 2001; 20: 4910-4915.

36. Grossman HB, Gomella L, Fradet Y, Morales A, Presti J, Ritenour C, Nseyo U, Droller MJ. A phase III, multicenter comparison of hexaminolevulinate fluorescence cystoscopy and white light cystoscopy for the detection of superficial papillary lesions in patients with bladder cancer. J Urol. 2007; 178: 62-67.

37. Fradet Y, Grossman HB, Gomella L, Lerner S, Cookson M, Albala D, Droller MJ. A comparison of hexaminolevulinate fluorescence cystoscopy and white light cystoscopy for the detection of carcinoma in situ in patients with bladder cancer: a phase III, multicenter study. J Urol. 2007; 178: 68-73.

38. Daniltchenko DI, Riedl CR, Sachs MD, Koenig F, Daha KL, Pflueger H, Loening SA, Schnorr D. Long-term benefit of 5-aminolevulinic acid fluorescence assisted transurethral resection of superficial bladder cancer: 5-year results of a prospective randomized study. J Urol. 2005; 174: 21292133.

39. Lee JY, Cho KS, Kang DH, Jung HD, Kwon JK, Oh CK, Ham WS, Choi YD. A network meta-analysis of therapeutic outcomes after new image technologyassisted transurethral resection for non-muscle invasive bladder cancer: 5-aminolaevulinic acid fluorescence vs hexylaminolevulinate fluorescence vs narrow band imaging. BMC Cancer. 2015; 15: 566.
40. Botteman MF, Pashos CL, Redaelli A, Laskin B, Hauser R. The health economics of bladder cancer. Pharmacoeconomics. 2003; 21: 1315-1330.

41. Avritscher EBC, Cooksley CD, Grossman HB, Sabichi AL, Hamblin L, Dinney CP, Elting LS. Clinical model of lifetime cost of treating bladder cancer and associated complications. Urology. 2006; 68: 549-553.

42. Cheung G, Sahai A, Billia M, Dasgupta P, Khan MS. Recent advances in the diagnosis and treatment of bladder cancer. BMC Med. 2013; 11: 1-8.

43. Grimbergen MCM, van Swol CFP, Jonges TGN, Boon TA, van Moorselaar RJA. Reduced specificity of 5-ALA induced fluorescence in photodynamic diagnosis of transitional cell carcinoma after previous intravesical therapy. Eur Urol. 2003; 44: 51-56.

44. Jocham D, Stepp H, Waidelich R. Photodynamic diagnosis in urology: state-of-the-art. Eur Urol. 2008; 53: 1138-1150.

45. Gono K, Obi T, Yamaguchi M, Ohyama N, Machida H, Sano Y, Yoshida S, Hamamoto Y, Endo T. Appearance of enhanced tissue features in narrow-band endoscopic imaging. J Biomed Opt. 2004; 9: 568-577.

46. Song PH, Cho S, Ko YH. Decision based on narrow band imaging cystoscopy without a referential normal standard rather increases unnecessary biopsy in detection of recurrent bladder urothelial carcinoma early after intravesical instillation. Cancer Res Treat. 2016; 48: 273-280.

47. Tatsugami K, Kuroiwa K, Kamoto T, Nishiyama H, Watanabe J, Ishikawa S, Shinohara N, Sazawa A, Fukushima S, Naito S. Evaluation of narrow-band imaging as a complementary method for the detection of bladder cancer. J Endourol. 2010; 24: 1807-1811. 